\title{
New Subclasses of Biunivalent Functions Involving Dziok-Srivastava Operator
}

\author{
M. K. Aouf, ${ }^{1}$ R. M. El-Ashwah, ${ }^{2}$ and Ahmed M. Abd-Eltawab ${ }^{3}$ \\ ${ }^{1}$ Department of Mathematics, Faculty of Science, Mansoura University, Mansoura 35516, Egypt \\ ${ }^{2}$ Department of Mathematics, Faculty of Science, Damietta University, New Damietta 34517, Egypt \\ ${ }^{3}$ Department of Mathematics, Faculty of Science, Fayoum University, Fayoum 63514, Egypt
}

Correspondence should be addressed to R. M. El-Ashwah; r_elashwah@yahoo.com

Received 23 June 2013; Accepted 15 July 2013

Academic Editors: R. Avery, D. Bahuguna, and Y. Han

Copyright (C) 2013 M. K. Aouf et al. This is an open access article distributed under the Creative Commons Attribution License, which permits unrestricted use, distribution, and reproduction in any medium, provided the original work is properly cited.

We introduce two new subclasses of biunivalent functions which are defined by using the Dziok-Srivastava operator. Furthermore, we find estimates on the coefficients $\left|a_{2}\right|$ and $\left|a_{3}\right|$ for functions in these new subclasses.

\section{Introduction}

Let $A$ denote the class of all functions of the form

$$
f(z)=z+\sum_{n=2}^{\infty} a_{n} z^{n}
$$

which are analytic in the open unit $\operatorname{disc} U=\{z \in \mathbb{C}:|z|<$ $1\}$. Also let $S$ denote the class of all functions in $A$ which are univalent in $U$.

Some of the important and well-investigated subclasses of the univalent function class $S$ include, for example, the class $S^{*}(\beta)$ of starlike functions of order $\beta$ in $U$ and the class $K(\beta)$ of convex functions of order $\beta$ in $U$. By definition, we have

$$
\begin{gathered}
S^{*}(\alpha)=\left\{f \in S: \operatorname{Re}\left(\frac{z f^{\prime}(z)}{f(z)}\right)>\beta,\right. \\
0 \leq \beta<1, z \in U\}, \\
K(\alpha)=\left\{f \in S: \operatorname{Re}\left(1+\frac{z f^{\prime \prime}(z)}{f^{\prime}(z)}\right)>\beta,\right. \\
0 \leq \beta<1, z \in U\} .
\end{gathered}
$$

Ding et al. [1] introduced the following class $Q_{\lambda}(\beta)$ of analytic functions defined as follows:

$$
\begin{array}{r}
Q_{\lambda}(\beta)=\left\{f \in A: \operatorname{Re}\left((1-\lambda) \frac{f(z)}{z}+\lambda f^{\prime}(z)\right)>\beta,\right. \\
0 \leq \beta<1, \lambda \geq 0\} .
\end{array}
$$

It is easy to see that $Q_{\lambda_{1}}(\beta) \subset Q_{\lambda_{2}}(\beta)$ for $\lambda_{1}>\lambda_{2} \geq 0$. Thus, for $\lambda \geq 1,0 \leq \beta<1, Q_{\lambda}(\beta) \subset Q_{1}(\beta)=\{f \in A$ : $\left.\operatorname{Re} f^{\prime}(z)>\beta, 0 \leq \beta<1\right\}$ and hence $Q_{\lambda}(\beta)$ is univalent class (see [2-4]).

It is well known that every function $f \in S$ has an inverse $f^{-1}$, defined by

$$
\begin{gathered}
f^{-1}(f(z))=z \quad(z \in U), \\
f\left(f^{-1}(w)\right)=w \quad\left(|w|<r_{0}(f) ; r_{0}(f) \geq \frac{1}{4}\right),
\end{gathered}
$$

where

$$
\begin{aligned}
f^{-1}(w)= & w-a_{2} w^{2}+\left(2 a_{2}^{2}-a_{3}\right) w^{3} \\
& -\left(5 a_{2}^{3}-5 a_{2} a_{3}+a_{4}\right) w_{4}+\cdots
\end{aligned}
$$

A function $f \in A$ is said to be bi-univalent in $U$ if both $f(z)$ and $f^{-1}(z)$ are univalent in $U$. Let $\Sigma$ denote the class of 
bi-univalent functions in $U$ given by (1). For a brief history and interesting examples in the class $\Sigma$ see [5].

Brannan and Taha [6] (see also [7]) introduced certain subclasses of the bi-univalent function class $\Sigma$ similar to the familiar subclasses $S^{*}(\beta)$ and $K(\beta)$ of starlike and convex functions of order $\beta(0 \leq \beta<1)$, respectively (see [8]). Thus, following Brannan and Taha [6] (see also [7]), a function $f \in A$ is in the class $S_{\Sigma}^{*}(\alpha)$ of strongly bi-starlike functions of order $\alpha(0<\alpha \leq 1)$ if each of the following conditions is satisfied:

$$
\begin{aligned}
& f \in \Sigma, \quad\left|\arg \left(\frac{z f^{\prime}(z)}{f(z)}\right)\right|<\frac{\alpha \pi}{2} \quad(0<\alpha \leq 1, z \in U), \\
& f \in \Sigma, \quad\left|\arg \left(\frac{z g^{\prime}(w)}{g(w)}\right)\right|<\frac{\alpha \pi}{2} \quad(0<\alpha \leq 1, z \in U),
\end{aligned}
$$

where $g$ is the extension of $f^{-1}$ to $U$. The classes $S_{\Sigma}^{*}(\alpha)$ and $K_{\Sigma}(\alpha)$ of bi-starlike functions of order $\alpha$ and biconvex functions of order $\alpha$, corresponding, respectively, to the function classes $S^{*}(\beta)$ and $K(\beta)$, were also introduced analogously. For each of the function classes $S_{\Sigma}^{*}(\alpha)$ and $K_{\Sigma}(\alpha)$, they found nonsharp estimates on the first two Taylor-Maclaurin coefficients $\left|a_{2}\right|$ and $\left|a_{3}\right|$ (for details, see $[6,7]$ ).

For function $f$ given by (1) and $g$ given by

$$
g(z)=z+\sum_{n=2}^{\infty} b_{n} z^{n}
$$

the Hadamard product (or convolution) of $f$ and $g$ is defined by

$$
(f * g)(z)=z+\sum_{n=2}^{\infty} a_{n} b_{n} z^{n}=(g * f)(z) .
$$

For complex parameters $a_{1}, \ldots, a_{q}$ and $b_{1}, \ldots, b_{s}(b j \notin$ $\left.\mathbb{Z}_{0}^{-}=\{0,-1,-2, \ldots\} ; j=1, \ldots, s\right)$, the generalized hypergeometric function ${ }_{q} F_{s}$ is defined by the following infinite series:

$$
\begin{gathered}
{ }_{q} F_{s}\left(a_{1}, \ldots, a_{q} ; b_{1}, \ldots, b_{s} ; z\right)=\sum_{n=0}^{\infty} \frac{\left(a_{1}\right)_{n} \cdots\left(a_{q}\right)_{n}}{\left(b_{1}\right)_{n} \cdots\left(b_{s}\right)_{n}} \frac{z^{n}}{n !} \\
\left(q \leq s+1 ; q, s \in \mathbb{N}_{0}=\mathbb{N} \cup\{0\}, \mathbb{N}=\{1,2,3, \ldots\} ; z \in U\right),
\end{gathered}
$$

where $(\theta)_{n}$ is the Pochhammer symbol (or shift factorial) defined, in terms of the Gamma function $\Gamma$, by

$$
(\theta)_{n}=\frac{\Gamma(\theta+n)}{\Gamma(\theta)}= \begin{cases}1, & (n=0) \\ \theta(\theta+1) \cdots(\theta+n-1), & (n \in \mathbb{N}) .\end{cases}
$$

Correspondingly a function $h\left(a_{1}, \ldots, a_{q} ; b_{1}, \ldots, b_{s} ; z\right)$ is defined by

$$
\begin{aligned}
& h\left(a_{1}, \ldots, a_{q} ; b_{1}, \ldots, b_{s} ; z\right) \\
& \quad=z_{q} F_{s}\left(a_{1}, \ldots, a_{q} ; b_{1}, \ldots, b_{s} ; z\right) \quad(z \in U) .
\end{aligned}
$$

Dziok and Srivastava [9] (see also [10]) considered a linear operator

$$
H\left(a_{1}, \ldots, a_{q} ; b_{1}, \ldots, b_{s}\right): A \longrightarrow A
$$

defined by the following Hadamard product:

$$
\begin{array}{r}
H\left(a_{1}, \ldots, a_{q} ; b_{1}, \ldots, b_{s}\right) f(z) \\
=h\left(a_{1}, \ldots, a_{q} ; b_{1}, \ldots, b_{s} ; z\right) * f(z), \\
\left(q \leq s+1 ; q, s \in \mathbb{N}_{0} ; z \in U\right) .
\end{array}
$$

If $f \in A$ is given by (1), then we have

$$
\begin{aligned}
& H\left(a_{1}, \ldots, a_{q} ; b_{1}, \ldots, b_{s}\right) f(z) \\
& \quad=z+\sum_{n=2}^{\infty} \Gamma_{n}\left[a_{1} ; b_{1}\right] a_{n} z^{n} \quad(z \in U),
\end{aligned}
$$

where

$$
\Gamma_{n}\left[a_{1} ; b_{1}\right]=\frac{\left(a_{1}\right)_{n} \cdots\left(a_{q}\right)_{n}}{\left(b_{1}\right)_{n} \cdots\left(b_{s}\right)_{n}} \frac{1}{n !} \quad(n \in \mathbb{N}) .
$$

To make the notation simple, we write

$$
H_{q, s}\left[a_{1} ; b_{1} ; z\right]=H\left(a_{1}, \ldots, a_{q} ; b_{1}, \ldots, b_{s}\right) f(z) .
$$

It easily follows from (14) that

$$
\begin{aligned}
& z\left(H_{q, s}\left[a_{1} ; b_{1} ; z\right]\right)^{\prime} \\
& \quad=a_{1} H_{q, s}\left[a_{1}+1 ; b_{1} ; z\right]-\left(a_{1}-1\right) H_{q, s}\left[a_{1} ; b_{1} ; z\right] .
\end{aligned}
$$

The linear operator $H_{q, s}\left[a_{1} ; b_{1} ; z\right]$ is a generalization of many other linear operators considered earlier.

The object of the present paper is to introduce two new subclasses of the bi-univalent functions which are defined by using the Dziok-Srivastava operator and find estimates on the coefficients $\left|a_{2}\right|$ and $\left|a_{3}\right|$ for functions in these new subclasses of the function class $\Sigma$ employing the techniques used earlier by Srivastava et al. [5] (see also [11]).

In order to derive our main results, we have to recall here the following lemma [12].

Lemma 1. If $h \in P$, then $\left|c_{k}\right| \leq 2$ for each $k$, where $P$ is the family of all functions $h$ analytic in $U$ for which $\operatorname{Re} h(z)>$ $0 h(z)=1+c_{1} z+c_{2} z^{2}+c_{3} z^{3}+\cdots$ for $z \in U$.

Unless otherwise mentioned, we assume throughout this paper that $a_{i}, b_{j} \in \mathbb{C} \backslash \mathbb{Z}_{0}^{-}, i=1, \ldots, s, j=1, \ldots, q, q \leq$ $s+1 ; q, s \in \mathbb{N}_{0}, 0<\alpha \leq 1, \lambda \geq 1, z \in U, \Gamma_{n}\left[a_{1} ; b_{1}\right]$ is given by (15) and all powers are understood as principle values. 


\section{Coefficient Bounds of the Function Class} $T_{q, s}^{\Sigma}\left[a_{1} ; b_{1}, \alpha, \lambda\right]$

Definition 2. One says that a function $f(z)$ given by (1) is said to be in the class $T_{q, s}^{\Sigma}\left[a_{1} ; b_{1}, \alpha, \lambda\right]$ if it satisfies the following condition:

$$
\begin{array}{r}
f \in \Sigma, \quad \mid \arg \left((1-\lambda) \frac{H_{q, s}\left[a_{1} ; b_{1} ; z\right]}{z}\right. \\
\left.+\lambda\left(H_{q, s}\left[a_{1} ; b_{1} ; z\right]\right)^{\prime}\right) \mid<\frac{\alpha \pi}{2}, \\
\left|\arg \left((1-\lambda) \frac{g(w)}{w}+\lambda g^{\prime}(w)\right)\right|<\frac{\alpha \pi}{2},
\end{array}
$$

where the function $g$ is given by

$$
\begin{aligned}
g(w)= & H_{q, s}^{-1}\left[a_{1} ; b_{1} ; z\right] \\
= & w-\Gamma_{2}\left[a_{1} ; b_{1}\right] a_{2} w^{2} \\
& +\left(2\left(\Gamma_{2}\left[a_{1} ; b_{1}\right]\right)^{2} a_{2}^{2}-\Gamma_{3}\left[a_{1} ; b_{1}\right] a_{3}\right) w^{3} \\
& -\left(5\left(\Gamma_{2}\left[a_{1} ; b_{1}\right]\right)^{3} a_{2}^{3}-5 \Gamma_{2}\left[a_{1} ; b_{1}\right]\right. \\
& \left.\times \Gamma_{3}\left[a_{1} ; b_{1}\right] a_{2} a_{3}+\Gamma_{4}\left[a_{1} ; b_{1}\right] a_{4}\right) w^{4}+\cdots
\end{aligned}
$$

Remark 3. (i) For $q=2, s=1$, and $a_{1}=a_{2}=b_{1}=1$, we have $T_{2,1}^{\Sigma}[1,1 ; 2 ; \alpha, \lambda]=B_{\Sigma}(\alpha, \lambda)$, where the class $B_{\Sigma}(\alpha, \lambda)$ was introduced and studied by Frasin and Aouf [11].

(ii) For $q=2, s=1$, and $a_{1}=a_{2}=b_{1}=\lambda=1$, we have $T_{2,1}^{\Sigma}[1,1 ; 2 ; \alpha, 1]=H_{\Sigma}(\alpha, \lambda)$, where the class $H_{\Sigma}(\alpha, \lambda)$ was introduced and studied by Srivastava et al. [5].

Theorem 4. Letting $f(z)$ given by (1) be in the class $T_{q, s}^{\Sigma}$ $\left[a_{1} ; b_{1}, \alpha, \lambda\right]$, then

$$
\begin{aligned}
\left|a_{2}\right| & =\frac{2 \alpha}{\left|\Gamma_{2}\left[a_{1} ; b_{1}\right]\right| \sqrt{(\lambda+1)^{2}+\alpha\left(1+2 \lambda-\lambda^{2}\right)}}, \\
\left|a_{3}\right| & =\frac{4 \alpha^{2}}{\left|\Gamma_{3}\left[a_{1} ; b_{1}\right]\right|(\lambda+1)^{2}}+\frac{2 \alpha}{\left|\Gamma_{3}\left[a_{1} ; b_{1}\right]\right|(2 \lambda+1)}
\end{aligned}
$$

Proof. It follows from (18) that

$$
\begin{gathered}
(1-\lambda) \frac{H_{q, s}\left[a_{1} ; b_{1} ; z\right]}{z}+\lambda\left(H_{q, s}\left[a_{1} ; b_{1} ; z\right]\right)^{\prime}=[p(z)]^{2}, \\
(1-\lambda) \frac{g(w)}{w}+\lambda g^{\prime}(w)=[q(w)]^{2},
\end{gathered}
$$

where $p(z)$ and $q(w)$ in $P$ have the forms

$$
\begin{aligned}
& p(z)=1+p_{1} z+p_{2} z^{2}+p_{3} z^{3}+\cdots, \\
& q(w)=1+q_{1} w+q_{2} w^{2}+q_{3} w^{3}+\cdots .
\end{aligned}
$$

Now, equating the coefficients in (22), we get

$$
\begin{gathered}
(\lambda+1) \Gamma_{2}\left[a_{1} ; b_{1}\right] a_{2}=\alpha p_{1}, \\
(2 \lambda+1) \Gamma_{3}\left[a_{1} ; b_{1}\right] a_{3}=\alpha p_{2}+\frac{\alpha(\alpha-1)}{2} p_{1}^{2}, \\
-(\lambda+1) \Gamma_{2}\left[a_{1} ; b_{1}\right] a_{2}=\alpha q_{1}, \\
(2 \lambda+1)\left(2\left(\Gamma_{2}\left[a_{1} ; b_{1}\right]\right)^{2} a_{2}^{2}-\Gamma_{3}\left[a_{1} ; b_{1}\right] a_{3}\right) \\
=\alpha q_{2}+\frac{\alpha(\alpha-1)}{2} q_{1}^{2} .
\end{gathered}
$$

From (25) and (27), we get

$$
\begin{gathered}
p_{1}=-q_{1}, \\
2(\lambda+1)^{2}\left(\Gamma_{2}\left[a_{1} ; b_{1}\right]\right)^{2} a_{2}^{2}=\alpha^{2}\left(p_{1}^{2}+q_{1}^{2}\right) .
\end{gathered}
$$

Now from (26), (28), and (30), we obtain

$$
\begin{aligned}
2(2 \lambda & +1)\left(\Gamma_{2}\left[a_{1} ; b_{1}\right]\right)^{2} a_{2}^{2} \\
& =\alpha\left(p_{2}+q_{2}\right)+\frac{\alpha(\alpha-1)}{2}\left(p_{1}^{2}+q_{1}^{2}\right) \\
& =\alpha\left(p_{2}+q_{2}\right)+\frac{\alpha(\alpha-1)}{2} \frac{2(\lambda+1)^{2}\left(\Gamma_{2}\left[a_{1} ; b_{1}\right]\right)^{2} a_{2}^{2}}{\alpha^{2}} .
\end{aligned}
$$

Therefore, we have

$$
a_{2}^{2}=\frac{\alpha^{2}\left(p_{2}+q_{2}\right)}{\left(\Gamma_{2}\left[a_{1} ; b_{1}\right]\right)^{2}\left[(\lambda+1)^{2}+\alpha\left(1+2 \lambda-\lambda^{2}\right)\right]} .
$$

Applying Lemma 1 for the coefficients $p_{2}$ and $q_{2}$, we immediately have

$$
\left|a_{2}\right| \leq \frac{2 \alpha}{\left|\Gamma_{2}\left[a_{1} ; b_{1}\right]\right| \sqrt{(\lambda+1)^{2}+\alpha\left(1+2 \lambda-\lambda^{2}\right)}} .
$$

This gives the bound on $\left|a_{2}\right|$ as asserted in (20).

Next, in order to find the bound on $\left|a_{3}\right|$, by subtracting (28) from (26) and using (29), we get

$$
\begin{aligned}
2(2 \lambda & +1) \Gamma_{3}\left[a_{1} ; b_{1}\right] a_{3}-2(2 \lambda+1)\left(\Gamma_{2}\left[a_{1} ; b_{1}\right]\right)^{2} a_{2}^{2} \\
& =\alpha p_{2}+\frac{\alpha(\alpha-1)}{2} p_{1}^{2}-\left(\alpha q_{2}+\frac{\alpha(\alpha-1)}{2} q_{1}^{2}\right) \\
& =\alpha\left(p_{2}-q_{2}\right) .
\end{aligned}
$$

It follows from (30) and (34) that

$$
\begin{aligned}
& 2(2 \lambda+1) \Gamma_{3}\left[a_{1} ; b_{1}\right] a_{3} \\
& \quad=\frac{\alpha^{2}(2 \lambda+1)\left(p_{1}^{2}+q_{1}^{2}\right)}{(\lambda+1)^{2}}+\alpha\left(p_{2}-q_{2}\right),
\end{aligned}
$$

And, then,

$$
a_{3}=\frac{\alpha^{2}\left(p_{1}^{2}+q_{1}^{2}\right)}{2(\lambda+1)^{2} \Gamma_{3}\left[a_{1} ; b_{1}\right]}+\frac{\alpha\left(p_{2}-q_{2}\right)}{2(2 \lambda+1) \Gamma_{3}\left[a_{1} ; b_{1}\right]} .
$$


Applying Lemma 1 once again for the coefficients $p_{1}, p_{2}, q_{1}$, and $q_{2}$, we readily get

$$
\left|a_{3}\right| \leq \frac{4 \alpha^{2}}{(\lambda+1)^{2}\left|\Gamma_{3}\left[a_{1} ; b_{1}\right]\right|}+\frac{2 \alpha}{(2 \lambda+1)\left|\Gamma_{3}\left[a_{1} ; b_{1}\right]\right|} .
$$

This completes the proof of Theorem 4.

Remark 5. (i) Taking $q=2, s=1$, and $a_{1}=a_{2}=b_{1}=1$, in Theorem 4, we obtain the result obtained by Frasin and Aouf [11, Theorem 2.2].

(ii) Taking $q=2, s=1$, and $a_{1}=a_{2}=b_{1}=\lambda=1$, in Theorem 4, we obtain the result obtained by Srivastava et al. [5, Theorem 1].

\section{Coefficient Bounds of the Function Class}

$$
T_{q, s}^{\Sigma}\left[a_{1} ; b_{1}, \beta, \lambda\right]
$$

Definition 6. One says that a function $f(z)$ given by (1) is said to be in the class $T_{q, s}^{\Sigma}\left[a_{1} ; b_{1}, \beta, \lambda\right]$ if it satisfies the following condition:

$$
\begin{gathered}
f \in \Sigma, \quad \operatorname{Re}\left\{(1-\lambda) \frac{H_{q, s}\left[a_{1} ; b_{1} ; z\right]}{z}\right. \\
\left.+\lambda\left(H_{q, s}\left[a_{1} ; b_{1} ; z\right]\right)^{\prime}\right\}>\beta, \\
\operatorname{Re}\left\{(1-\lambda) \frac{g(w)}{w}+\lambda g^{\prime}(w)\right\}>\beta,
\end{gathered}
$$

where the function $g$ is defined by (19).

Remark 7. (i) For $q=2, s=1$, and $a_{1}=a_{2}=b_{1}=1$, we have $T_{2,1}^{\Sigma}[1,1 ; 2 ; \beta, \lambda]=B_{\Sigma}(\beta, \lambda)$, where the class $B_{\Sigma}(\beta, \lambda)$ was introduced and studied by Frasin and Aouf [11].

(ii) For $q=2, s=1$, and $a_{1}=a_{2}=b_{1}=\lambda=1$, we have $T_{2,1}^{\Sigma}[1,1 ; 2 ; \beta, 1]=H_{\Sigma}(\beta, \lambda)$, where the class $H_{\Sigma}(\beta, \lambda)$ was introduced and studied by Srivastava et al. [5].

Theorem 8. Letting $f(z)$ given by (1) be in the class $T_{q, s}^{\Sigma}$ $\left[a_{1} ; b_{1}, \beta, \lambda\right], 0 \leq \beta<1$ and $\lambda \geq 1$, then

$$
\begin{gathered}
\left|a_{2}\right|=\frac{\sqrt{2(1-\beta)}}{\left|\Gamma_{2}\left[a_{1} ; b_{1}\right]\right| \sqrt{2 \lambda+1}}, \\
\left|a_{3}\right|=\frac{4(1-\beta)^{2}}{\left|\Gamma_{3}\left[a_{1} ; b_{1}\right]\right|(\lambda+1)^{2}}+\frac{2(1-\beta)}{\left|\Gamma_{3}\left[a_{1} ; b_{1}\right]\right|(2 \lambda+1)} .
\end{gathered}
$$

Proof. It follows from (38) that

$$
\begin{aligned}
& (1-\lambda) \frac{H_{q, s}\left[a_{1} ; b_{1} ; z\right]}{z}+\lambda\left(H_{q, s}\left[a_{1} ; b_{1} ; z\right]\right)^{\prime} \\
& =\beta+(1-\beta) p(z), \\
& (1-\lambda) \frac{g(w)}{w}+\lambda g^{\prime}(w)=\beta+(1-\beta) q(w),
\end{aligned}
$$

where $p(z)$ and $q(w)$ have the forms (23) and (24), respectively.

As in the proof of Theorem 4, by suitably comparing coefficients in (41), we get

$$
\begin{gathered}
(\lambda+1) \Gamma_{2}\left[a_{1} ; b_{1}\right] a_{2}=(1-\beta) p_{1}, \\
(2 \lambda+1) \Gamma_{3}\left[a_{1} ; b_{1}\right] a_{3}=(1-\beta) p_{2}, \\
-(\lambda+1) \Gamma_{2}\left[a_{1} ; b_{1}\right] a_{2}=(1-\beta) q_{1}, \\
(2 \lambda+1)\left(2\left(\Gamma_{2}\left[a_{1} ; b_{1}\right]\right)^{2} a_{2}^{2}-\Gamma_{3}\left[a_{1} ; b_{1}\right] a_{3}\right)=(1-\beta) q_{2} .
\end{gathered}
$$

From (42) and (44), we get

$$
\begin{gathered}
p_{1}=-q_{1}, \\
2(\lambda+1)^{2}\left(\Gamma_{2}\left[a_{1} ; b_{1}\right]\right)^{2} a_{2}^{2}=(1-\beta)^{2}\left(p_{1}^{2}+q_{1}^{2}\right) .
\end{gathered}
$$

Also, from (43) and (45), we find that

$$
2(2 \lambda+1)\left(\Gamma_{2}\left[a_{1} ; b_{1}\right]\right)^{2} a_{2}^{2}=(1-\beta)\left(p_{2}+q_{2}\right) .
$$

Therefore, we have

$$
\left|a_{2}^{2}\right| \leq \frac{(1-\beta)}{\left(\Gamma_{2}\left[a_{1} ; b_{1}\right]\right)^{2}[2(2 \lambda+1)]}\left(\left|p_{2}\right|+\left|q_{2}\right|\right) .
$$

Applying Lemma 1 for the coefficients $p_{2}$ and $q_{2}$, we immediately have

$$
\left|a_{2}\right| \leq \frac{\sqrt{2(1-\beta)}}{\left|\Gamma_{2}\left[a_{1} ; b_{1}\right]\right| \sqrt{2 \lambda+1}} .
$$

This gives the bound on $\left|a_{2}\right|$ as asserted in (39).

Next, in order to find the bound on $\left|a_{3}\right|$, by subtracting (45) from (43), we get

$$
\begin{aligned}
2(2 \lambda & +1) \Gamma_{3}\left[a_{1} ; b_{1}\right] a_{3}-2(2 \lambda+1)\left(\Gamma_{2}\left[a_{1} ; b_{1}\right]\right)^{2} a_{2}^{2} \\
& =(1-\beta)\left(p_{2}-q_{2}\right),
\end{aligned}
$$

or, equivalently,

$$
a_{3}=\frac{\left(\Gamma_{2}\left[a_{1} ; b_{1}\right]\right)^{2} a_{2}^{2}}{\Gamma_{3}\left[a_{1} ; b_{1}\right]}+\frac{(1-\beta)\left(p_{2}-q_{2}\right)}{2(2 \lambda+1) \Gamma_{3}\left[a_{1} ; b_{1}\right]},
$$

and, then from (47), we find that

$$
a_{3}=\frac{(1-\beta)^{2}\left(p_{1}^{2}+q_{1}^{2}\right)}{2(\lambda+1)^{2} \Gamma_{3}\left[a_{1} ; b_{1}\right]}+\frac{(1-\beta)\left(p_{2}-q_{2}\right)}{2(2 \lambda+1) \Gamma_{3}\left[a_{1} ; b_{1}\right]} .
$$

Applying Lemma 1 once again for the coefficients $p_{1}, p_{2}, q_{1}$, and $q_{2}$, we readily get

$$
\left|a_{3}\right| \leq \frac{4(1-\beta)^{2}}{(\lambda+1)^{2}\left|\Gamma_{3}\left[a_{1} ; b_{1}\right]\right|}+\frac{2(1-\beta)}{(2 \lambda+1)\left|\Gamma_{3}\left[a_{1} ; b_{1}\right]\right|} .
$$

This completes the proof of Theorem 8 . 
Remark 9. (i) Taking $q=2, s=1$, and $a_{1}=a_{2}=b_{1}=1$, in Theorem 8, we obtain the result obtained by Frasin and Aouf [11, Theorem 3.2].

(ii) Taking $q=2, s=1$, and $a_{1}=a_{2}=b_{1}=\lambda=1$, in Theorem 8 , we obtain the result obtained by Srivastava et al. [5, Theorem 2].

\section{References}

[1] S. S. Ding, Y. Ling, and G. J. Bao, "Some properties of a class of analytic functions," Journal of Mathematical Analysis and Applications, vol. 195, no. 1, pp. 71-81, 1995.

[2] M. P. Chen, "On the regular functions satisfying $(f(z) / z)>\alpha$," Bulletin of the Institute of Mathematics. Academia Sinica, vol. 3, no. 1, pp. 65-70, 1975.

[3] P. N. Chichra, "New subclasses of the class of close-to-convex functions," Proceedings of the American Mathematical Society, vol. 62, no. 1, pp. 37-43, 1976.

[4] T. H. MacGregor, "Functions whose derivative has a positive real part," Transactions of the American Mathematical Society, vol. 104, pp. 532-537, 1962.

[5] H. M. Srivastava, A. K. Mishra, and P. Gochhayat, "Certain subclasses of analytic and bi-univalent functions," Applied Mathematics Letters, vol. 23, no. 10, pp. 1188-1192, 2010.

[6] D. A. Brannan and T. S. Taha, "On some classes of bi-univalent functions," in Mathematical Analysis and Its Applications, S. M. Mazhar, A. Hamoui, and N. S. Faour, Eds., vol. 3 of KFAS Proceedings Series, pp. 53-60, Pergamon Press, Oxford, UK, 1985, see also Studia Universitatis Babeş-Bolyai. Series Mathematica, vol. 31, no. 2, pp. 70-77, 1986.

[7] T. S. Taha, Topics in univalent function theory [Ph.D. thesis], University of London, London, UK, 1981.

[8] D. A. Brannan, J. Clunie, and W. E. Kirwan, "Coefficient estimates for a class of star-like functions," Canadian Journal of Mathematics, vol. 22, pp. 476-485, 1970.

[9] J. Dziok and H. M. Srivastava, "Classes of analytic functions associated with the generalized hypergeometric function," Applied Mathematics and Computation, vol. 103, no. 1, pp. 1-13, 1999.

[10] J. Dziok and H. M. Srivastava, "Certain subclasses of analytic functions associated with the generalized hypergeometric function," Integral Transforms and Special Functions, vol. 14, no. 1, pp. 7-18, 2003.

[11] B. A. Frasin and M. K. Aouf, "New subclasses of bi-univalent functions," Applied Mathematics Letters, vol. 24, no. 9, pp. 15691573, 2011.

[12] C. Pommerenke, Univalent Functions, Vandenhoeck \& Ruprecht, Göttingen, Germany, 1975. 


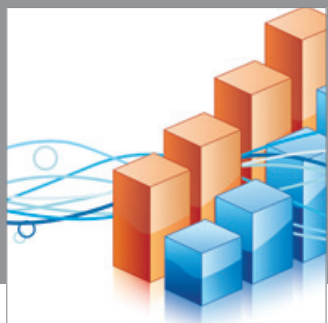

Advances in

Operations Research

mansans

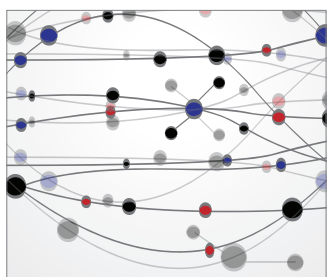

The Scientific World Journal
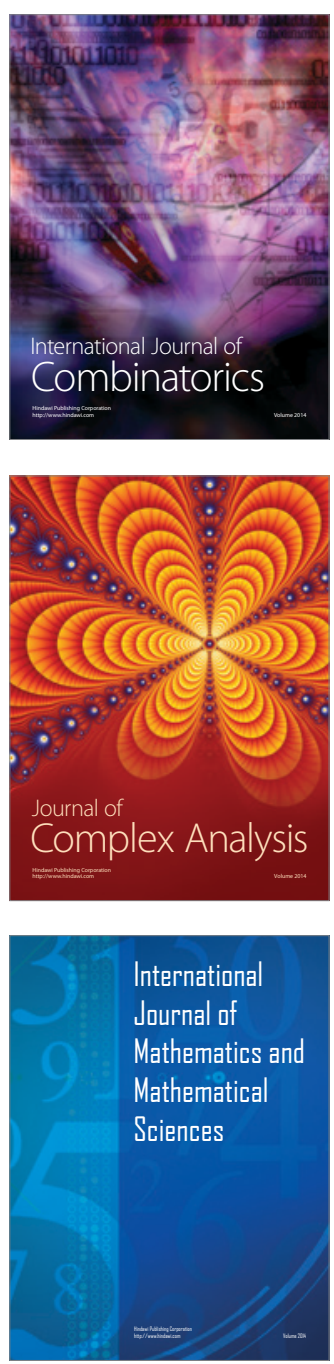
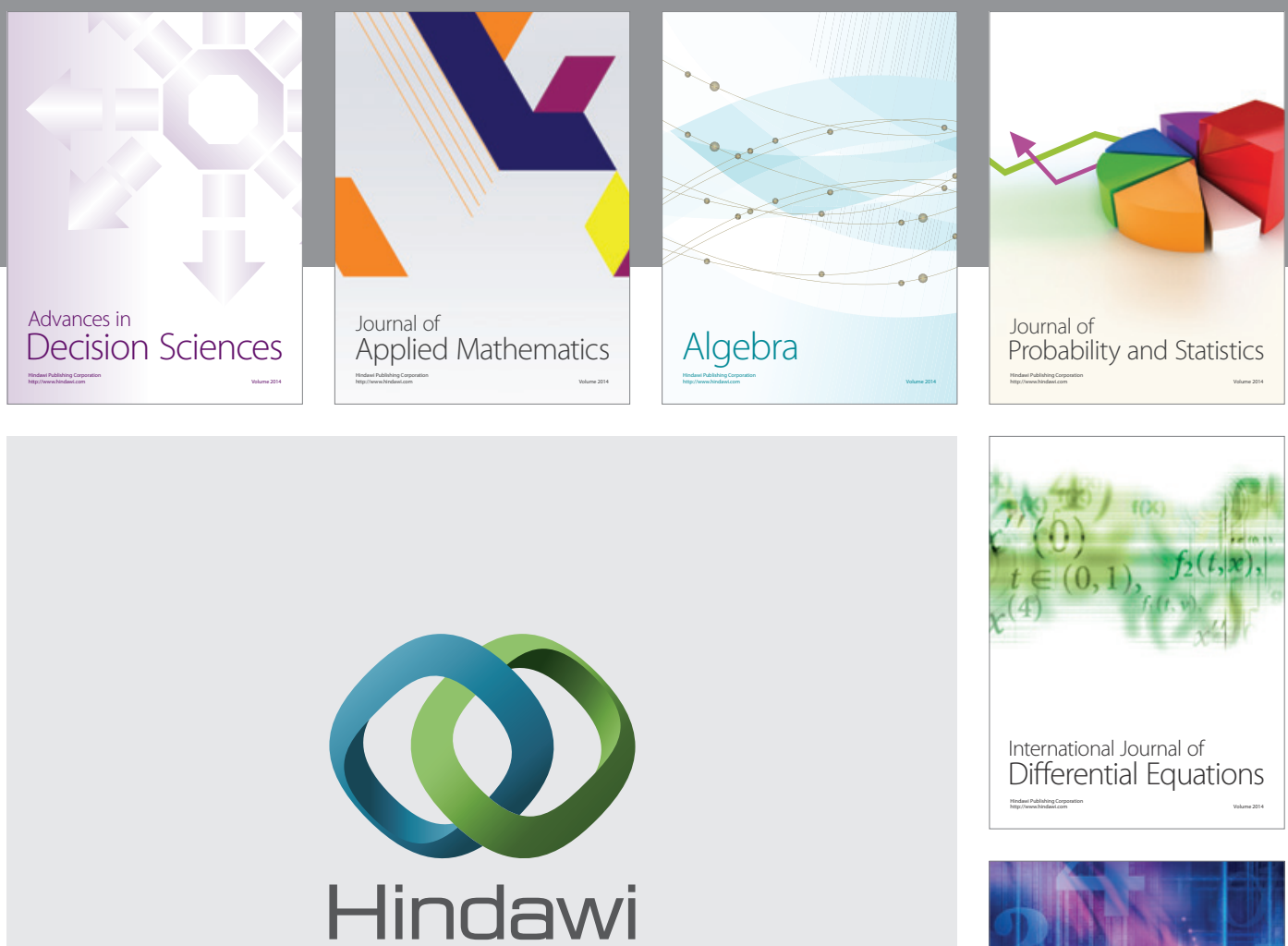

Submit your manuscripts at http://www.hindawi.com
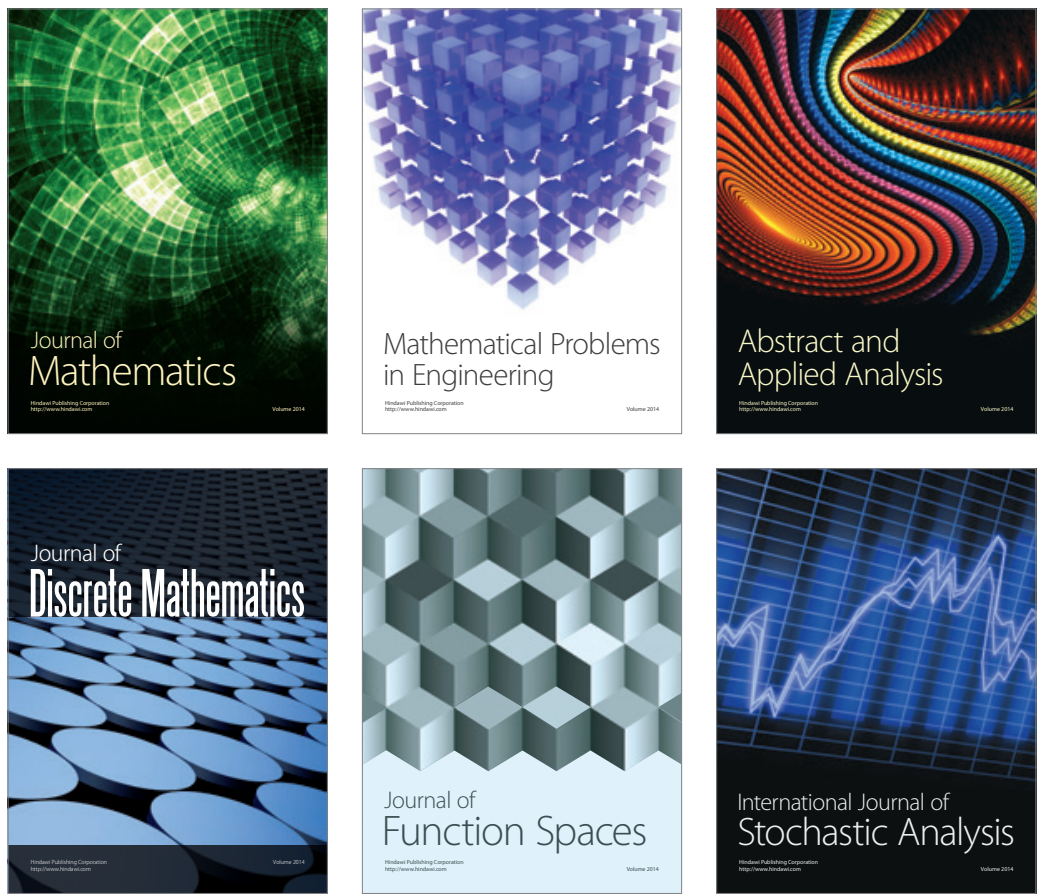

Journal of

Function Spaces

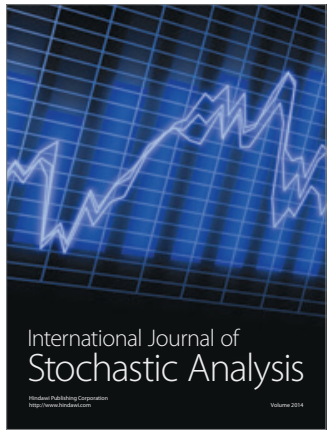

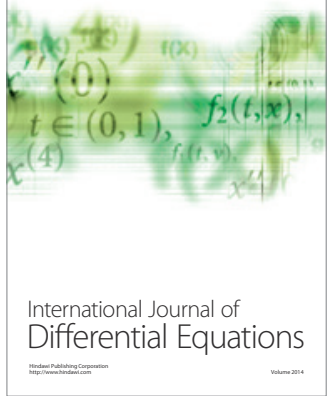
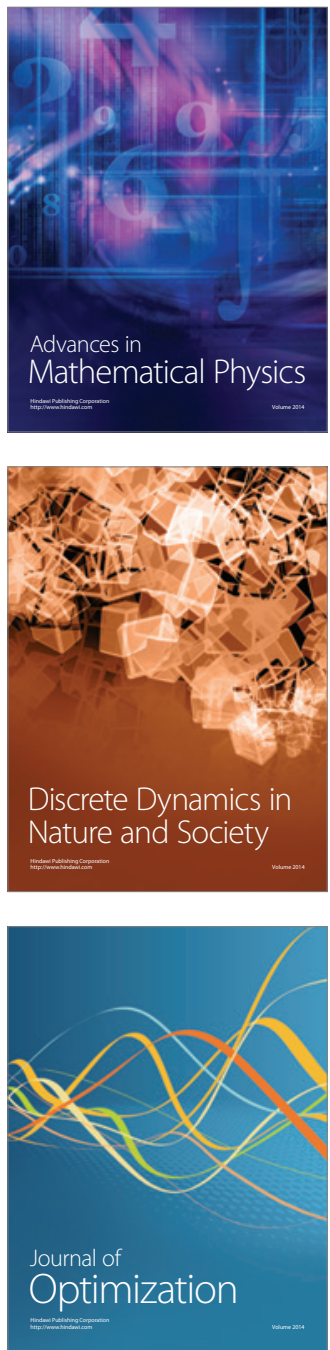